\title{
Non-relativistic String Theory
}

\section{Eric A. Bergshoeff*}

Van Swinderen Institute

E-mail: E.A.Bergshoeff@rug.nI

Johannes Lahnsteiner

Van Swinderen Institute

E-mail: jo.lahnsteiner@hotmail.com

\section{Luca Romano}

Van Swinderen Institute

E-mail: lucaromano2607@gmail.com

\section{Ceyda Şimşek}

Van Swinderen Institute

E-mail: c.simsek@rug.nl

We show how Newton-Cartan gravity can be generalized to String Newton-Cartan gravity which is identified as the proper geometry underlying non-relativistic string theory. Specific properties of non-relativistic string theory are discussed such as T-duality and (solutions to) the equations of motion of the background fields.

Corfu Summer Institute 2019 "School and Workshops on Elementary Particle Physics and Gravity" (CORFU2019)

31 August - 25 September 2019

Corfù, Greece

${ }^{*}$ Speaker. 


\section{Introduction}

Starting from classical mechanics, there are at least three interesting ways to extend the theory each of which introduces a constant of nature that is absent in classical mechanics: (1) at large velocities with respect to the velocity of light $c$ the theory extends to special relativity; (2) at small distances certain physical quantities get quantized in units of the reduced Planck's constant $\hbar$ corresponding to quantum mechanics and (3) a gravitational force can be introduced via Newton's constant $G$ leading to Newtonian gravity. There are two well-known ways to combine two of these extensions: (1) extending classical mechanics with high velocities and gravity leads to general relativity and (2) extending classical mechanics to high velocities and small distances leads to quantum field theory. Logically speaking, however, there is a third way, namely extending classical mechanics to small distances and gravity. This would lead to a theory of non-relativistic (NR) quantum gravity. Finally, the maximal extension to high velocities, small distances and gravity leads to the long looked for theory of quantum gravity, see the so-called Bronstein cube [1] in Figure 1.

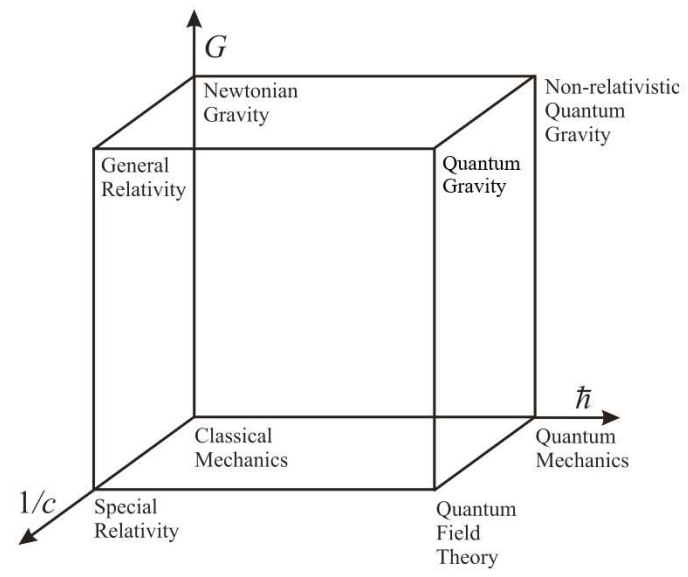

Figure 1: The Bronstein cube shows how classical mechanics can be extended in three different ways to (1) special relativity, (2) quantum mechanics and (3) Newtonian gravity. Combining two of these extensions leads to general relativity, quantum field theory or NR quantum gravity. Ultimately, combining all three extensions leads to quantum gravity.

Usually, the issue of finding a consistent theory of quantum gravity is approached either by adding gravity to quantum field theory or by quantizing general relativity. The Bronstein cube suggests a third way to approach this issue: can quantum gravity be viewed as the relativistic extension of a self-consistent NR theory of quantum gravity? This leads to the related question of how essential relativity is in constructing a theory of quantum gravity or, put differently, whether one can take in a consistent way the NR limit of quantum gravity. Motivated by this we wish to address the following intriguing question:

$$
\text { can one define a consistent NR theory of quantum gravity? }
$$

String theory is one approach to define a theory of quantum gravity. In this talk we wish to 
discuss the definition of a NR string theory including its underlying geometry and some of its basic properties. We will first show how the geometry corresponding to NR string theory can be viewed as a generalization of the well-known Newton-Cartan (NC) geometry that underlies NC gravity.

\section{From NC Gravity to String NC Gravity}

The independent fields of $D$-dimensional NC geometry are given by $(a=1, \cdots, D-1)$

$$
\left\{\tau_{\mu}, E_{\mu}{ }^{a}, M_{\mu}\right\}
$$

Here, $\tau_{\mu}$ is the time-like Vierbein acting as the clock function and $E_{\mu}{ }^{a}$ is the spatial Vierbein acting as the ruler. The charge corresponding to the gauge field $M_{\mu}$ is a central charge in the Galilei algebra thereby extending it to the Bargmann algebra. These gauge fields transform under (local) spatial rotations with parameters $\lambda^{a}{ }_{b}$, Galilean boosts with parameters $\lambda^{a}$ and central charge transformations with parameter $\sigma$ as follows:

$$
\begin{aligned}
\delta \tau_{\mu} & =0, \\
\delta E_{\mu}{ }^{a} & =\lambda^{a}{ }_{b} E_{\mu}{ }^{b}+\lambda^{a} \tau_{\mu}, \\
\delta M_{\mu} & =\partial_{\mu} \sigma+\lambda_{a} E_{\mu}{ }^{a} .
\end{aligned}
$$

The spin-connection fields $\Omega_{\mu}{ }^{a b}$ corresponding to spatial rotations and $\Omega_{\mu}{ }^{a}$ corresponding to Galilean boosts are functions of $\tau_{\mu}, E_{\mu}{ }^{a}$ and $M_{\mu}$.

In NC gravity one cannot define a single non-degenerate metric for the full spacetime like the Riemannian metric in general relativity. Instead, one can define two degenerate metrics

$$
\tau_{\mu v}=\tau_{\mu} \tau_{v} \quad \text { and } \quad h^{\mu v}=E^{\mu}{ }_{a} E_{b}^{v} \delta^{a b}
$$

that are invariant under the Bargmann transformations (2.2). Here $E^{\mu}{ }_{a}$ is the projective inverse of $E_{\mu}{ }^{a}$ which, unlike the spatial Vierbein, is invariant under Galilean boosts. This means that the combination

$$
E_{\mu}^{a} E_{v}^{b} \delta_{a b}
$$

is not invariant under Galilean boosts and, for this reason, cannot be used as a metric. In order to make a boost-invariant combination one often considers the combination

$$
H_{\mu v}=E_{\mu}{ }^{a} E_{v}{ }^{b} \delta_{a b}+M_{\mu} \tau_{v}+M_{v} \tau_{\mu}
$$

However, this combination is not invariant under central charge transformations. Nevertheless, it is used in the construction of a NR particle action coupled to NC gravity in such a way that the central charge gauge field $M_{\mu}$ couples to the particle via a Wess-Zumino (WZ) term of the form

$$
M_{\mu} \dot{x}^{\mu}
$$

where $x^{\mu}(\tau)$ is an embedding coordinate. This leads to a particle Lagrangian that is invariant under central charge transformations up to a total derivative. We will often call the symmetric tensor $H_{\mu \nu}$ the transverse metric and $\tau_{\mu \nu}$ the longitudinal metric. 
The central charge gauge field $M_{\mu}$ of NC gravity has a precursor in general relativity as an Abelian gauge field $\hat{M}_{\mu}$ to be added to general relativity. The only difference is that the Poincaré algebra does not get modified by the gauge field $\hat{M}_{\mu}$. This gauge field plays a crucial role in constructing NR limits without divergencies. For instance, starting from the Klein-Gordon Lagrangian coupled to general relativity one can only obtain the Schrödinger Lagrangian coupled to NC gravity as a NR limit provided one extends general relativity with a fluxless Abelian gauge field $\hat{M}_{\mu}$ that couples to a complex Klein-Gordon scalar. Similarly, one can only define a NR limit of a relativistic particle coupled to general relativity without divergencies provided the relativistic particle couples to $\hat{M}_{\mu}$ via a WZ term of the form

$$
\hat{M}_{\mu} \dot{x}^{\mu}
$$

It is instructive to give some details here. To define the NR limit we first express the Riemannian metric of general relativity and the gauge field $\hat{M}_{\mu}$ in terms of the NC fields (2.1) and a contraction parameter $\omega$. Next, after substituting these expressions into the action of the relativistic particle coupled to general relativity, we take the limit $\omega \rightarrow \infty$. This leads to a divergence linear in $\omega$ coming form the kinetic term that is cancelled by a similar divergent term coming from the WZ term by expressing $\hat{M}_{\mu}$ in terms of the NC fields as follows:

$$
\hat{M}_{\mu}=\omega \tau_{\mu}+m_{\mu} .
$$

Given the fact that a vector field only couples via a WZ term to a particle, it is clear that one cannot apply the same procedure to define the NR limit of a string. In this case, it is the KalbRamond 2-form gauge field $\hat{B}_{\mu \nu}$ that couples to the relativistic string via a WZ term of the form

$$
\varepsilon^{\alpha \beta} \partial_{\alpha} x^{\mu} \partial_{\beta} x^{v} \hat{B}_{\mu v}
$$

where $\partial_{\alpha}(\alpha=0,1)$ is the derivative with respect to the world-sheet coordinates $\sigma^{\alpha}$ and $x^{\mu}\left(\sigma^{\alpha}\right)$ are the string embedding coordinates. It turns out that taking the NR limit of a string leads to a divergence quadratic in $\omega$ coming from the kinetic term. To cancel this quadratic divergence we cannot work with a NC geometry since that contains only one clock function $\tau_{\mu}$ and there is no way to express the Kalb-Ramond field in terms of this single clock function. To cancel the quadratic divergence coming from the kinetic term we need two clock functions $\tau_{\mu}{ }^{A}(A=0,1)$ and write

$$
\hat{B}_{\mu v}=\omega^{2} \varepsilon_{A B} \tau_{\mu}{ }^{A} \tau_{v}{ }^{B}+B_{\mu \nu},
$$

where $B_{\mu \nu}$ is the NR Kalb-Ramond field. This leads to a new so-called SNC geometry that is characterized by two special directions instead of the single Newtonian time direction in NC gravity. The difference between particles and strings is that a particle sweeps out a one-dimensional time direction whereas a sting sweeps out two directions longitudinal to the string: one time direction and one spatial direction, see Figure 2.

Ignoring central extensions the algebra underlying the SNC geometry is the so-called string Galilei algebra where we distinguish between the two directions $A=0,1$ longitudinal to the string and the remaining directions $a=2, \cdots D-1$ transverse to the string. We thus have

$$
D \text { flat indices } \rightarrow\left\{\begin{array}{l}
2 \text { longitudinal indices A } \\
\text { D-2 transverse indices a }
\end{array}\right.
$$




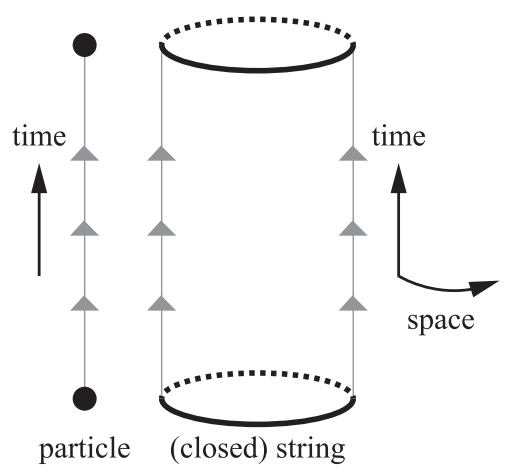

Figure 2: A particle (left) sweeps out a one-dimensional time direction whereas a string (right) sweeps out two directions: one time and one spatial direction.

with the following symmetries and generators:

$$
\begin{array}{rc}
\text { longitudinal translations } & H_{A} \\
\text { transverse translations } & P_{a} \\
\text { string Galilei boosts } & G_{A b} \\
\text { longitudinal Lorentz rotations } & M_{A B} \\
\text { transverse spatial rotations } & J_{a b}
\end{array}
$$

This string Galilei algebra is extended to a so-called enhanced string Galilei algebra with two types of non-central ${ }^{1}$ generators:

$$
Z_{A} \quad \text { and } \quad Z_{A B} \text { with } Z_{A}^{A}=0 .
$$

The independent string NC fields are

$$
\left\{\tau_{\mu}{ }^{A}, E_{\mu}{ }^{a}, M_{\mu}{ }^{A}\right\}
$$

For the construction of a NR string action we need both a longitudinal metric $\tau_{\mu v}$ and a transverse metric $H_{\mu \nu}$ which are the following generalizations of the particle case given in eqs. (2.3) and (2.5), respectively:

$$
\begin{aligned}
\text { longitudinal metric: } & \tau_{\mu \nu} \equiv \tau_{\mu}{ }^{A} \tau_{v}{ }^{B} \eta_{A B} \\
\text { transverse metric: } & H_{\mu v} \equiv E_{\mu}{ }^{a} E_{v}{ }^{b} \delta_{a b}+\left(\tau_{\mu}{ }^{A} M_{v}{ }^{B}+\tau_{v}{ }^{A} M_{\mu}{ }^{B}\right) \eta_{A B}
\end{aligned}
$$

\section{Non-relativistic String Theory}

We are now in a position to construct the action of NR string theory in a general SNC gravity background. For flat spacetime the action was already given a long time ago and reads $[2,3]$

$$
S_{\text {flat }}=-\frac{1}{4 \pi \alpha^{\prime}} \int d^{2} \sigma\left(\partial x^{a} \bar{\partial} x^{b} \delta_{a b}+\lambda \bar{\partial} X+\bar{\lambda} \partial \bar{X}\right)
$$

\footnotetext{
${ }^{1}$ We call a generator non-central if it only has non-zero commutators due to its index structure.
} 
with

$$
X=x^{0}+x^{1}, \quad \bar{X}=x^{0}-x^{1}
$$

and similar for the Lagrange multipliers $\lambda, \bar{\lambda}$. A special feature of NR string theory is that the (perturbative) spectrum only contains winding strings along the compact $x^{1}$ direction [2].

The presence of the Lagrange multipliers can be understood as the result of taking the NR limit of the relativistic string action in Polyakov form. This is best explained by considering the following relativistic particle action coupled to general relativity in Polyakov form:

$$
S_{\text {Pol. }}=-\frac{1}{2} \int d \tau\left\{-\frac{1}{e} \hat{E}_{\mu} \hat{A}^{\mu} \hat{E}_{v} \hat{B}^{v} \eta_{\hat{A} \hat{B}}+M^{2} e-2 M \hat{M}_{\mu} \dot{x}^{\mu}\right\} .
$$

Here $e$ is the worldline Einbein and $M$ is a mass parameter. Expanding the general relativity fields in terms of the SNC background fields one encounters the following quadratic divergence that is not cancelled by the Kalb-Ramond field in the Wess-Zumino term:

$$
S_{\text {Pol. }}\left(\omega^{2}\right)=-\frac{1}{2} \int d \tau \frac{1}{e} \omega^{2}\left[\tau_{\mu} \dot{x}^{\mu}-m e\right]^{2} .
$$

It should be noted that this is an artefact of the Polyakov formulation. In the Nambu-Goto formulation there is no quadratic divergence left. The quadratic divergence given in (3.3) is not fatal. The reason for this is that it is the square of something and therefore can be re-written, using a Lagrange multiplier $\lambda$ as follows:

$$
S_{\text {Pol. }}\left(\omega^{2}\right)=-\frac{1}{2} \int d \tau \frac{1}{e}\left\{\lambda\left(\tau_{\mu} \dot{x}^{\mu}-m e\right)-\frac{1}{4 \omega^{2}} \lambda^{2}\right\}
$$

Written in this form, the limit that $\omega \rightarrow \infty$ can be taken and one ends up with the following NR Polyakov action:

$$
S_{\text {Pol. }}(\text { N.R. })=-\frac{1}{2} \int d \tau \frac{1}{e}\left\{\dot{x}^{\mu} \dot{x}^{v} H_{\mu v}+\lambda\left(\tau_{\mu} \dot{x}^{\mu}-m e\right)\right\} .
$$

Integrating out the Lagrange multiplier $\lambda$ one finds that

$$
e=\frac{\tau_{\mu} \dot{x}^{\mu}}{m}
$$

Substituting this back into the Polyakov action (3.5) one obtains the following NR particle action in Nambu-Goto form:

$$
S_{\text {N.G. }}(\text { N.R. })=-\frac{m}{2} \int d \tau \frac{\dot{x}^{\mu} \dot{x}^{v}}{\tau_{\rho} \dot{x}^{\rho}} H_{\mu \nu} .
$$

One can now take a similar limit of the relativistic Polyakov string. We thus find the following expression for a NR string in a (matter-coupled) SNC background $[4,5]:^{2}$

$$
\begin{aligned}
S_{\mathrm{SNC}}= & -\frac{T}{2} \int d^{2} \sigma\left[\sqrt{-h} h^{\alpha \beta} \partial_{\alpha} x^{\mu} \partial_{\beta} x^{v} H_{\mu v}+\varepsilon^{\alpha \beta}\left(\lambda e_{\alpha} \tau_{\mu}+\bar{\lambda} \bar{e}_{\alpha} \bar{\tau}_{\mu}\right) \partial_{\beta} x^{\mu}\right] \\
& -\frac{T}{2} \int d^{2} \sigma \varepsilon^{\alpha \beta} \partial_{\alpha} x^{\mu} \partial_{\beta} x^{v} B_{\mu v}+\frac{1}{4 \pi} \int d^{2} \sigma \sqrt{-h} R\left(\Phi-\frac{1}{4} \ln G\right),
\end{aligned}
$$

\footnotetext{
${ }^{2}$ For other recent work on non-relativistic strings in a curved background, see $[6,7,8,9,10,11,12]$.
} 
where $T$ is the string tension, $\sigma^{\alpha}$ are the world-sheet coordinates, $h_{\alpha \beta}=e_{\alpha}{ }^{a} e_{\beta}{ }^{b} \eta_{a b}$ is the worldsheet metric with Zweibeine $e_{\alpha}{ }^{a}, R^{(2)}$ is the Ricci scalar defined with respect to $h_{\alpha \beta}$ and $x^{\mu}(\sigma)$, $\mu=0,1, \cdots, D-1$ are the string embedding coordinates. The action (3.8) also describes the coupling to the background Kalb-Ramond field $B_{\mu \nu}$ and the dilaton $\Phi$. Furthermore, $\lambda$ and $\bar{\lambda}$ are two world-sheet Lagrange multiplier fields whose equations of motion allow us to solve for the world-sheet metric $h_{\alpha \beta}$ in terms of the pullback of the longitudinal metric $\tau_{\mu \nu}$ as follows:

$$
h_{\alpha \beta}=\partial_{\alpha} x^{\mu} \partial_{\beta} x^{v} \tau_{\mu v} .
$$

The definition of $G$ occurring in the string sigma model action (3.8) in terms of $H_{\mu \nu}$ and $\tau_{\mu}{ }^{A}$ is given by

$$
G=\operatorname{det} H_{\mu \nu} \operatorname{det}\left(\tau_{\rho}^{A} H^{\rho \sigma} \tau_{\sigma}^{B}\right) .
$$

Finally, the lightcone components $\tau_{\mu}, \bar{\tau}_{\mu}$ of $\tau_{\mu}{ }^{A}$ and $e_{\alpha}, \bar{e}_{\alpha}$ of $e_{\alpha}{ }^{a}$ are defined in [4,5].

Upon integrating out the Lagrange multipliers, one can show that the string action is invariant under Galilean boosts with parameters $\lambda^{A A^{\prime}}$, non-central charge transformations with parameters $\lambda^{A}$ and second non-central charge transformations with parameters $\sigma_{B}^{A}\left(\right.$ with $\sigma_{A}^{A}=0$ ):

$$
\begin{aligned}
\delta \tau_{\mu}{ }^{A} & =0, \\
\delta E_{\mu}{ }^{A^{\prime}} & =-\lambda_{A}{ }^{A^{\prime}} \tau_{\mu}{ }^{A}, \\
\delta m_{\mu}{ }^{A} & =D_{\mu}(\omega) \lambda^{A}+\lambda^{A}{ }_{A^{\prime}} E_{\mu}{ }^{A^{\prime}}+\sigma^{A}{ }_{B} \tau_{\mu}{ }^{B} .
\end{aligned}
$$

Here $D_{\mu}(\omega)$ is the Lorentz-covariant derivative with respect to the longitudinal Lorentz rotations. Note that the gauge field corresponding to the second non-central charge transformation does not occur in the string action. The invariance under the first non-central charge transformations is valid provided that the following zero torsion constraint holds: ${ }^{3}$

$$
D_{[\mu}(\omega) \tau_{v]}^{A}=0
$$

Part of this constraint contains the spin-connection field $\omega_{\mu}{ }^{A B}$, enabling one to solve this connection field in terms of $\tau_{\mu}{ }^{A}$ and its derivative. The remaining part is a geometric constraint given by the projection of (3.12) that does not contain the spin-connection:

$$
\varepsilon_{C}^{\left({ }^{A}\right.} \tau_{[\mu}^{B)} \partial_{v} \tau_{\rho]}^{C}=0
$$

An important feature of the NR action (3.8), which is absent in the relativistic case, is that the action is invariant under certain Stückelberg symmetries of the background fields implying that some of the components only occur in special combinations. A similar thing happens for the NR Nambu-Goto particle coupled to a vector gauge field $B_{\mu}$ :

$$
S_{\mathrm{NG}}(\text { N.R. })=-\frac{m}{2} \int d \tau\left\{\frac{\dot{x}^{\mu} \dot{x}^{v}}{\tau_{\rho} \dot{x}^{\rho}} H_{\mu v}-B_{\mu} \dot{x}^{\mu}\right\},
$$

\footnotetext{
${ }^{3}$ At the classical level there is another way to achieve invariance of the action under the first non-central charge transformations by assigning to the Kalb-Ramond field an extra central charge transformation that is proportional to the torsion [12].
} 
in which case the Stïckelberg symmetries are given by

$$
H_{\mu v} \rightarrow H_{\mu v}+\frac{1}{2}\left(\tau_{\mu} C_{v}+\tau_{v} C_{\mu}\right), \quad B_{\mu} \rightarrow C_{\mu} .
$$

In terms of the Stückelberg-invariant combinations the NR particle action (3.14) reads

$$
S_{\mathrm{NG}}(\mathrm{N} . \mathrm{R} .)=-\frac{m}{2} \int d \tau\left\{\frac{E^{A^{\prime}} E^{B^{\prime}} \delta_{A^{\prime} B^{\prime}}}{\tau}+\tau\left(H_{00}-B_{0}\right)+E^{A^{\prime}}\left(H_{0 A^{\prime}}-B_{A^{\prime}}\right)\right\}
$$

where we have used flat indices and where we have defined

$$
\tau \equiv \dot{x}^{\mu} \tau_{\mu}, \quad E^{A^{\prime}} \equiv \dot{x}^{\mu} E_{\mu}{ }^{A \prime}
$$

Similarly, one finds that the NR string action (3.8) is invariant under the following Stückelberg symmetries

$$
\begin{gathered}
H_{\mu v} \rightarrow H_{\mu v}-\left(C_{\mu}{ }^{A} \tau_{v}^{B}+C_{v}{ }^{A} \tau_{\mu}{ }^{B}\right) \eta_{A B}, \\
B_{\mu v} \rightarrow B_{\mu v}+\left(C_{\mu}{ }^{A} \tau_{v}^{B}-C_{v}{ }^{A} \tau_{\mu}{ }^{B}\right) \varepsilon_{A B}
\end{gathered}
$$

in terms of an arbitrary parameter $C_{\mu}{ }^{A}(x)$.

\section{T-duality}

Relativistic T-duality relates relativistic string theory compactified on a small radius to string theory compactified on a large radius. It is a perturbative symmetry that can also relate different string theories. T-duality is a stringy phenomenon, but, as we will see in this section, it does not depend on the relativistic nature of string theory. For the convenience of the reader, we will first briefly review the relativistic case since the procedure to derive the NR T-duality rules is very similar. Our starting point is the relativistic Polyakov string action given by

$$
\begin{aligned}
S=- & \frac{T}{2} \int d^{2} \sigma\left\{\sqrt{\gamma} \gamma^{\alpha \beta} \partial_{\alpha} x^{\mu} \partial_{\beta} x^{v} \hat{G}_{\mu v}+\varepsilon^{\alpha \beta} \partial_{\alpha} x^{\mu} \partial_{\beta} x^{v} \hat{B}_{\mu v}+\right. \\
& \left.+\frac{1}{4 \pi} \int d^{2} \sigma \sqrt{-h} R\left(\hat{\Phi}-\frac{1}{4} \ln (-\hat{G})\right)\right\},
\end{aligned}
$$

where $\hat{G}_{\mu \nu}$ is the spacetime metric, $\hat{B}_{\mu \nu}$ is the Kalb-Ramond two-form, $\hat{\Phi}$ is the dilaton field and $\hat{G}=\operatorname{det} \hat{G}_{\mu \nu}$. We split the embedding coordinates as $x^{\mu}=\left(y, x^{i}\right)$ where $y$ is a spacelike coordinate and make a corresponding decomposition of the spacetime fields in the action (4.1). We then assume that $y$ is an isometry direction and that the background fields are independent of $y$. Next, we replace $\partial_{\alpha} y$ by $V_{\alpha}$ introducing a Lagrange multiplier $\tilde{y}$ that inforces the following equation of motion of $V_{\alpha}$ :

$$
\varepsilon^{\alpha \beta} \partial_{\alpha} V_{\beta}=0
$$

One can solve (4.2) as $V_{\beta}=\partial_{\beta} y$ (ignoring possible non-contractible cycles on the worldsheet) and substitute this solution back into the action to obtain the original action (4.1). 
Instead, one can solve for the equation of motion of $V_{\alpha}$ as

$$
V^{\alpha}=\frac{1}{\hat{G}_{y y}}\left(\hat{G}_{y i} \partial_{\beta} x^{i} \eta^{\alpha \beta}+\varepsilon^{\alpha \beta} \hat{B}_{y i} \partial_{\beta} x^{i}+\varepsilon^{\alpha \beta} \partial_{\beta} \tilde{y}\right)
$$

and substitute this solution back into the action. The resulting action describes a relativistic string dual sigma model in a dual background. ${ }^{4}$ The map between the background fields specified by $\hat{G}_{\mu \nu}, \hat{B}_{\mu \nu}$ and $\hat{\Phi}$ as in eq. (4.1) and the dual background fields $\tilde{G}_{\mu \nu}, \tilde{B}_{\mu \nu}$ and $\tilde{\Phi}$ is given by the socalled Buscher rules $[13,14]$. The fact that these two backgrounds are T-dual to each other implies that the original string sigma model (4.1), when reduced along a spatial target space direction, gives the same theory that one obtains after a (dual) spacelike reduction of the dual string sigma model. The T-duality transformation can also be used as a solution generating transformation in the sense that the T-dual of a solution of the background field equation of motion produces a new solution of the same set of equations of motion.

Now, we would like to perform the procedure described above to obtain the nonrelativistic T-duality rules. ${ }^{5}$ The basic difference with the relativistic case is that in NR string theory the longitudinal and transverse directions are not at the same footing. In the action (3.8), this asymmetry is manifest in the terms with the Lagrange multipliers

$$
\varepsilon^{\alpha \beta}\left(\lambda e_{\alpha} \tau_{\mu}+\bar{\lambda} \bar{e}_{\alpha} \bar{\tau}_{\mu}\right)
$$

where the string only couples to the longitudinal vierbein $\tau_{\mu}, \bar{\tau}_{\mu}$. This explains why there are distinct T-dualities depending on the nature of the isometry direction. Here, we summarize the results of three different T-dualities characterized by the isometry direction $y$ :

i. Longitudinal Spatial T-duality relates NR string theory on a SNC background to relativistic string theory on a Riemannian background with a null isometry $K=\partial_{y}$ :

$$
\left(\tau_{\mu}{ }^{A}, H_{\mu \nu}, B_{\mu \nu}, \Phi\right) \stackrel{T}{\longrightarrow}\left(\tilde{G}_{\mu \nu}, \tilde{B}_{\mu \nu}, \tilde{\Phi}\right) \text { with } \quad \tilde{G}_{y y}=0 .
$$

Note that the longitudinal spatial isometry becomes null in the dual theory. There are fewer fields in the NR string theory than in the relativistic string theory due to the presence of the Stuickelberg symmetries (3.18) in the NR case.

ii. Longitudinal Lightlike T-duality maps the NR string theory on a SNC background with a longitudinal lightlike isometry to NR string theory on a T-dual SNC background.

iii. Transverse T-duality maps NR string theory on a SNC geometry with a transverse isometry to a NR string theory on a dual SNC background. The longitudinal Vierbein $\tau_{\mu}{ }^{A}$ is inert under this type of T-duality. The SNC fields transform to the dual SNC fields in a way that is very similar to the Buscher's rules in the relativistic case.

\footnotetext{
${ }^{4}$ The equation of motion of $V^{\alpha}$ does not determine how the dilaton field transforms. Its transformation is obtained to compensate the shift in $\hat{G}$ under T-duality transformations. In the non-relativistic string theory, one has to take into account the contribution of the Lagrange multipliers to the determinant $\hat{G}$ if they are integrated out [4].

${ }^{5}$ In nonrelativistic string theory, one can derive the analogue of the Buscher rules in two ways. One either applies the description above to the NR sigma model (3.8) [4] or takes a nonrelativistic limit of the Buscher rules [5]. These two approaches produce the same results for the longitudinal T-duality and the transverse T-duality. However, if the isometry direction is lightlike, the NR limit of the Buscher rules is singular.
} 
Like in relativistic string theory, NR T-duality can be used as a tool to generate solutions of NR string theory. Remarkably, the longitudinal T-duality allows us to produce solutions of NR string theory by taking the T-dual of relativistic string solutions equipped with a null isometry. There is one restriction in this scenario. The non-relativistic sigma model requires the geometric constraints (3.13); therefore, the relativistic solution should also satisfy the T-dual of these geometric constraints. In the next section we will give an explicit example of how to construct out of a known solution of general relativity a new solution of SNC gravity.

\section{Equations of Motion}

The equations of motion of the SNC background fields can be obtained by taking the NR limit of the relativistic beta functions. Our starting point is the set of relativistic string theory beta functions which up to $O\left(\alpha^{\prime 2}\right)$ are given by

$$
\begin{aligned}
& \beta_{\mu \nu}^{\hat{G}}=\alpha^{\prime}\left(\hat{R}_{\mu v}+2 \hat{\nabla}_{\mu} \hat{\nabla}_{v} \hat{\Phi}-\frac{1}{4} \hat{H}_{\mu \rho \sigma} \hat{H}_{v}^{\rho \sigma}\right), \\
& \beta_{\mu v}^{\hat{B}}=\alpha^{\prime}\left(-\frac{1}{2} \hat{\nabla}^{\rho} \hat{H}_{\rho \mu \nu}+\hat{\nabla}^{\rho} \hat{\Phi} \hat{H}_{\rho \mu \nu}\right), \\
& \beta^{\hat{F}}=\frac{D-26}{2}-\alpha^{\prime}\left(\hat{\nabla}_{\mu} \hat{\nabla}^{\mu} \hat{\Phi}+\frac{1}{4} \hat{R}-\hat{\nabla}_{\mu} \hat{\Phi}^{\mu} \hat{\nabla}^{\mu} \hat{\Phi}-\frac{1}{48} \hat{H}_{\mu \nu \rho} \hat{H}^{\mu v \rho}\right),
\end{aligned}
$$

where

$$
\begin{aligned}
\hat{H}_{\mu v \rho} & =\partial_{\mu} \hat{B}_{v \rho}+\partial_{v} \hat{B}_{\rho \mu}+\partial_{\rho} \hat{B}_{v \mu} \\
\hat{F} & =\hat{\Phi}-\frac{1}{4} \ln (-\hat{G})
\end{aligned}
$$

Following $[15,16,5]$, making the following expansion in inverse powers of $c$,

$$
\begin{aligned}
\hat{\Gamma}_{\mu \nu}^{\rho} & =\Gamma_{\mu v}^{\rho}+O\left(\omega^{-2}\right), \\
\hat{R}_{\sigma \mu v}^{\rho} & =R_{\sigma \mu v}^{\rho}+O\left(\omega^{-2}\right), \\
\hat{H}_{\mu v \rho} & =H_{\mu v \rho}+O\left(\omega^{-2}\right), \\
H_{\mu v \rho} & =\partial_{\mu} B_{v \rho}+\partial_{v} B_{\rho \mu}+\partial_{\rho} B_{v \mu},
\end{aligned}
$$

it is possible to take the $\omega \rightarrow \infty$ limit of the equations above and the result defines the equations of motion of SNC gravity coupled to the B field and dilaton, with the further condition given by the 
geometric constraints (3.13). We thus find, up to $O\left(\alpha^{\prime 2}\right)$, the following SNC equations [5]

$$
\begin{gathered}
E_{A^{\prime}}^{\mu} E_{B^{\prime}}^{v} \beta_{\mu \nu}^{H}=\alpha^{\prime} P_{A^{\prime} B^{\prime}}, \\
E_{A^{\prime}}^{\mu} E_{B^{\prime}}^{v} \beta_{\mu \nu}^{B}=\alpha^{\prime} Q_{A^{\prime} B^{\prime}}, \\
\tau_{A}^{\mu} \tau_{B}^{v}\left(\eta^{A B} \beta_{\mu \nu}^{H}-\varepsilon^{A B} \beta_{\mu \nu}^{B}\right)=\alpha^{\prime}\left(\eta^{A B} P_{A B}-\varepsilon^{A B} Q_{A B}\right), \\
\tau_{B}^{\mu} E_{A^{\prime}}^{v}\left(\delta_{A}^{B} \beta_{\mu \nu}^{H}+\varepsilon_{A}^{B} \beta_{\mu \nu}^{B}\right)=\alpha^{\prime}\left(P_{A A^{\prime}}+\varepsilon_{A}^{B} Q_{B A^{\prime}}\right), \\
\beta^{F}=\frac{D-26}{2}-\alpha^{\prime}\left(\nabla_{A^{\prime}} \nabla^{A^{\prime}} \Phi+\frac{1}{4} R_{A^{\prime}}^{A^{\prime}}-\nabla_{A^{\prime}} \Phi \nabla^{A^{\prime}} \Phi-\frac{1}{48} H_{A^{\prime} B^{\prime} C^{\prime}} H^{A^{\prime} B^{\prime} C^{\prime}}\right),
\end{gathered}
$$

where

$$
\begin{aligned}
P_{\mu v} & =R_{\mu v}+2 \nabla_{\mu} \nabla_{v} \Phi-\frac{1}{4} H_{\mu \rho \sigma} H_{v}{ }^{\rho \sigma}, \\
Q_{\mu v} & =-\frac{1}{2} \nabla^{A^{\prime}} H_{A^{\prime} \mu \nu}+\nabla^{A^{\prime}} \Phi H_{A^{\prime} \mu v} .
\end{aligned}
$$

Note that, due to the Stückelberg symmetries (3.18), there are no equations of motion solely in the longitudinal directions except for the single equation given in eq. (5.4c)

We now wish to construct solutions to these SNC equations of motion by applying the NR T-duality rules, discussed in the previous section, to solutions of the DSM. Acceptable solutions of the DSM are much easier to find by taking a solution of general relativity that has a null isometry direction and that satisfies the T-dual of the geometric constraints (3.13). A first interesting example is given by the $p p$-wave solution of general relativity: ${ }^{6}$

$$
\begin{aligned}
d s^{2} & =2 d u d v+K\left(z_{(8)}\right) d u^{2}+d z_{(8)}^{2}, \\
\hat{B} & =0, \\
e^{\hat{\phi}} & =g_{s},
\end{aligned}
$$

where $x^{\mu}=\left\{u, v, z_{(8)}\right\}, u, v$ are two null-directions and $K\left(_{(8)}\right)$ is a harmonic function of the transverse coordinates $z_{(8)}$. This solution has a null-isometry direction $v$ and, as it turns out, satisfies the T-dual of the geometric constraints (3.13). Thus, it fulfills all the requirements to be a solution of the DSM. We can therefore obtain a solution of SNC gravity by acting on this pp-wave with an (inverse) NR T-duality along the null isometry direction $v$. In this way we obtain the following NR fundamental string solution of SNC gravity:

$$
\begin{aligned}
\tau_{\mu}^{0} d x^{\mu} & =d x^{0}, \\
\tau_{\mu}{ }^{1} d x^{\mu} & =d y, \\
H_{\mu \nu} d x^{\mu} d x^{\nu} & =K\left(z_{(8)}\right)\left(d x^{\underline{0}}\right)^{2}+d z_{(8)}^{2}, \\
B_{\mu \nu} & =0, \\
e^{\phi} & =g_{s},
\end{aligned}
$$

\footnotetext{
${ }^{6}$ Actually, this solution is a $u$-independent truncation of a $p p$-wave solution.
} 
where $x^{\mu}=\left\{x^{0}, y, z_{(8)}\right\}$ and $y$ is the image under the inverse map of the $v$ direction defining the spatial direction along the fundamental string. Taking $D=10$, a null reduction of the $p p$-wave solution generates a 0-brane solution in nine dimensions. From a T-dual point of view, the same 9D 0-brane solution can be obtained by a dimensional reduction of the NR fundamental string solution along the longitudinal spatial direction, see Figure 3.

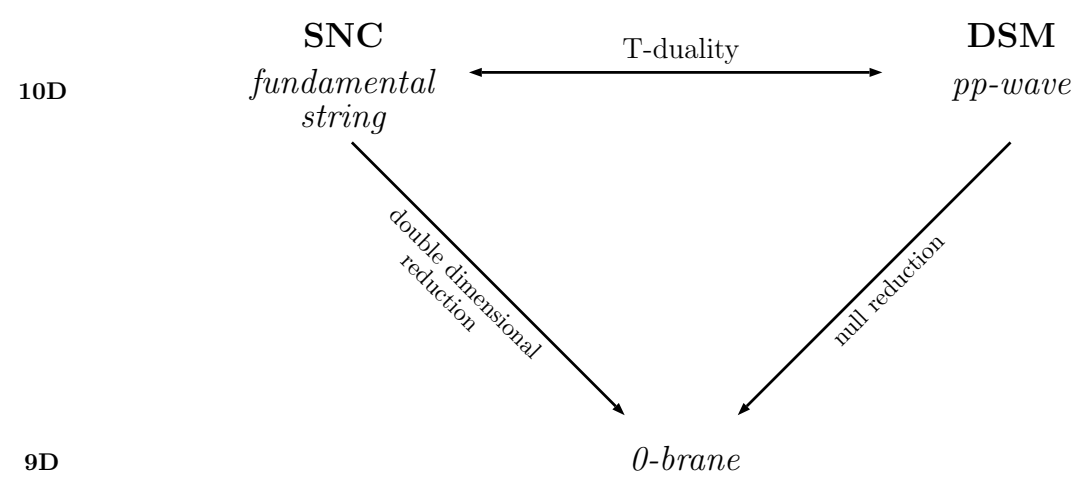

Figure 3: The $p p$-wave solution in the DSM is mapped under T-duality along a null isometry direction to the fundamental string solution in SNC gravity. This implies that a null reduction of the $p p$-wave leads to the same nine dimensional 0-brane solution as the one obtained by a double dimensional reduction of the SNC fundamental string.

In the NR solution that we have obtained not all the components of the SNC fields are specified. This signals the fact that the NR string only couples to a subset of the (matter coupled) SNC fields, i.e. the string only couples to those combinations of fields that are invariant under the Stuickelberg symmetries given in eq. (3.18). We furthermore note that, opposite to the relativistic case, the NR fundamental string solution has a zero dilaton. This was to be expected from the T-dual point of view: the NR fundamental string is the image under T-duality of the $p p$-wave in the DSM and, according to Figure 3, this $p p$-wave should reduce to the same 9D 0-brane solution as the fundamental string. However, the reduction of the $p p$-wave along a null isometric direction $v$ produces a zero dilaton since the corresponding Kaluza-Klein scalar $G_{v v}$ is identically zero in the DSM. This explains why also the fundamental string solution has a zero dilaton.

These and other particular properties of the fundamental string and the existence of more solutions will be discussed in more detail in a forthcoming publication.

\section{Conclusions}

In this talk we showed how NC geometry, which is the natural arena for NR particles, should be extended to what we called SNC geometry in order to incorporate the notion of NR strings. In fact, we gave the explicit expression for the Polyakov form of a NR string moving in a general SNC background [4, 5] thereby extending the case of a flat background [2,3] and of special curved backgrounds [17]. We gave both the action and the symmetries that form the underlying SNC algebra. A special feature is that there are additional Stückelberg symmetries which are related to the fact that not all components of the (matter coupled) SNC gravity couple to the string. As an example of this phenomenon we discussed the NR particle where a similar thing happens. 
After presenting the NR string action we discussed how the relativistic T-duality could be extended to a NR T-duality. A crucial difference with the relativistic case is that there exist different T-dualities depending on the direction of the isometry direction with respect to the directions longitudinal to the string. We discussed three different kinds of NR T-dualities.

We finally discussed the equations of motion of the (matter coupled) SNC background fields and showed how the NR T-duality can be used to produce out of known solutions of general relativity that satisfy certain restrictions new solutions of SNC gravity. As an example we gave the $p p$-wave solution of general relativity that gave us the so-called fundamental string solution of SNC gravity.

We hope that further investigations of the NR string theory presented in this talk will finally answer the question whether the vertex in the Bronstein cube, see Figure 1, representing NR quantum gravity can be given a meaning independent of relativity theory and, in particular, whether there exist an independent notion of non-relativistic holography.

\section{Acknowledgements}

This talk was based upon the papers $[4,5]$. We thank our collaborators for the many stimulating discussions we had with them. We also thank the organizers for a very stimulating atmosphere.

\section{References}

[1] Bronstein M 1933 Uspekhi Astronomicheskikh Nauk. Sbornik 3, 3-30 ; Stache J, in: Ciufolini I, Dominici D and Lusanna L(eds) 2001 A Relativistic Spacetime Odyssey World Scientific (2003)

[2] Gomis J and Ooguri H 2001 Nonrelativistic closed string theory J. Math. Phys. 423127

[3] Danielsson U.H., Guijosa A and Kruczenski M, IIA/B, wound and wrapped 2000 JHEP 0010020

[4] Bergshoeff E, Gomis J and Yan Z Nonrelativistic String Theory and T-Duality JHEP 1811 (2018) 133

[5] Bergshoeff E A, Gomis J, Rosseel J, Şimşek C and Yan Z String Theory and String Newton-Cartan Geometry J. Phys. A 53 (2020) no.1, 014001

[6] Harmark T, Hartong J and Obers N A 2017 Nonrelativistic strings and limits of the AdS/CFT correspondence, Phys. Rev. D 96 no.8, 086019

[7] Klusoň J 2018 Remark About Non-Relativistic String in Newton-Cartan Background and Null Reduction, JHEP 1805041

[8] Harmark T, Hartong J, Menculini L, Obers N A and Yan, Z 2018 Strings with Non-Relativistic Conformal Symmetry and Limits of the AdS/CFT Correspondence, JHEP 1811190

[9] Klusoň J 2019 Note About T-duality of Non-Relativistic String JHEP 1908074

[10] Klusoň J 2019 (m,n)-String and D1-Brane in Stringy Newton-Cartan Background, JHEP 1904163

[11] Roychowdhury D 2019 Probing tachyon kinks in Newton-Cartan background, Phys. Lett. B 795225

[12] Harmark T, Hartong J, Menculini L, Obers N A and Oling G 2019 Relating non-relativistic string theories JHEP 1911071

[13] Buscher T H Path Integral Derivation of Quantum Duality in Nonlinear Sigma Models 1988 Phys. Lett. B 201466. 
[14] Buscher T H A Symmetry of the String Background Field Equations 1987 Phys. Lett. B 194, 59.

[15] Gomis J, Oh J and Yan Z 2019 Nonrelativistic String Theory in Background Fields JHEP 1910101

[16] Yan Z and Yu M Background Field Method for Nonlinear Sigma Models in Nonrelativistic String Theory, arXiv:1912.03181 [hep-th].

[17] Gomis J, Gomis J and Kamimura M 2005 Non-relativistic superstrings: A New soluble sector of $\operatorname{AdS}(5)$ x $S^{5}$ JHEP 0512024 